Fournal of Information Technology (1994) 9, 71-72

\title{
Editorial
}

\section{THEME ISSUE - ORGANIZATIONAL PERSPECTIVES ON COLLABORATIVE WORKING}

\author{
THEME EDITORS: Chris Clegg, Department of Psychology, University of Sheffield \\ and Leslie Willcocks, Templeton College, University of Oxford
}

\section{Papers on Collaborative Working}

It is a pleasure this quarter to devote a substantial part of the issue to papers from an organization studies perspective. It is particularly important to apply such a perspective to the field under study, routinely called Computer-Supported Cooperative Work (CSCW). This is, firstly, because the number of applications of this type is rapidly growing. Secondly, all too often in IS development and implementation, despite several decades of literature advising against technology-led practice, the main emphasis is placed on the 'computer-supported' part of the term CSCW. To further reorientate attention we have also chosen to replace the word 'cooperative' with the more generic descriptor 'collaborative'. The former word suggests a unitary perspective on how and why people work together. The latter term implies that people may also actually differ in their perceptions and the objectives and interests they pursue at work in their use of technologies. We see it as important that a focus on human and organizational issues should also be fully informed and alive to the differing organization studies-based perspectives that have been, and can be, brought to bear on IS developments in work organizations. The following papers put flesh on this guideline for our theme issue.

John McCarthy provides our introduction with a review of the 'state-of-the-art' of CSCW. He points out the understandable diversity in definition and approach in what is a relatively young field. In particular he usefully pinpoints tensions between theoretical positions and statements of intent on the one hand and CSCW research and design on the other. He critically analyses the nature and sources of these tensions through addressing three questions: To what extent is CSCW technology-led? What kind of understanding of work is reflected in CSCW systems and research? To what extent does CSCW have or represent an organizational perspective? He concludes his review with some suggestions for ways forward.

Chris Clegg, Pat Waterson and Neil Carey add to the picture firstly by detailing their research on implementation of advanced manufacturing technologies and office automation systems. They find that, in these application domains, development and implementation have been technology-led, and have largely excluded a range of psychological and organizational concerns. Two further cases - of CSCW this time, with CADCAM and Information Engineering systems - show a similar pattern. They delineate the contributory social factors that help to explain why such a pattern is routinely repeated and derive a number of lessons for those working in the CSCW field as researchers or practitioners. Their findings take on even deeper significance if a basic premiss of Chris Hutchison and Duska Rosenberg is correct: that the emerging new breed if IS will have a more significant impact on how people work than existing, more 'conventional' IT-based products. The particular issue they wish to focus on is changes in the 'cognitive style' of user interaction. As they put it: "one does not "converse" with an expert system in the way ones does with, say, a spreadsheet, just as one does not "walk through" a multimedia "virtual museum" or a wide-area information server in the way one navigates a conventional database'. They then provide a view of the organization of organizations. This looks at the ontological status of organizations and the related concepts of process, office, culture, structure and role. They then provide a view on cooperation and conflict in CSCW, and discuss the role of language in organizational behaviour and IS design.

The contribution of Judi Ellis and Yvonne Rogers is to provide a new framework for CSCW capable of capturing cognitive activities as embodied and situated within the social and organizational context in which they occur. Previous frameworks have been partial, because each has been developed from within a particular research group or discipline, for example sociology, cognitive psychology, or anthropology. The distributed cognition framework is adopted from the work of Hutchins and his colleagues at the University of California. The unit of analysis adopted is the collection of individuals and artefacts in the work setting. The focus is on the relations and interactions between the individuals and artefacts. The authors describe the approach in detail and how it can be operationalized. They then provide two case studies they have analysed using the distributed cognition framework 
- the cases cover computer mediated work in an engineering practice, and coordination of activity in a hospital department.

Frank Blackler provides yet another perspective on $\mathrm{CSCW}$, this time through the lens of recent developments in social theory and organizational analysis. Firstly he critiques the CSCW literature where it provides a strong rhetoric supporting the notion that the future will be determined by technological developments. Secondly, however, he argues that developments in global capitalism, including developments in networking technologies, are sufficiently unusual as to merit a reevaluation of the way in which groups and organizations can be analysed. The author then contrasts modernist with postmodernist approaches, and suggests that the latter provide useful ways of thinking about organizations which "as they become independent of geographical location, devoid of conventional hierarchy, and with no obvious boundaries between themselves and other organizations, are becoming increasingly difficult to understand". Blackler's call for reviewing the intellectual context for understanding CSCW, and his focus on the postmodern, provides a fitting paper to end a theme issue devoted to developing further how computer supported collaborative working can be studied.

\section{Other papers}

The remaining papers that make up this issue focus on three different areas. Andrew Friedman revists the Nolan stage model of growth and develops the concept of the IS field. He then suggests that so far there have been three phases of the IS field: hardware capacity constraints, software productivity constraints and user relation constraints. Friedman then compares his phases model with that of Nolan's and updates the latter into eight stages. Of particular interest here is the new Stage 6, called strategic systems representing present growth in IS expenditure on EDI, VANs and systems for which the organization's customers and suppliers are direct end users.

E. Reed Doke and Tonya Barrier set themselves another classificatory task. They review previous taxonomies of information systems and argue that these are inadequate for describing all of today's type of IS. They then propose a new taxonomy based on the system user and support dimensions. The taxonomy is illustrated with reference to modern systems. In the final article Alan Sangster reports on a UK survey into the extent to which expert systems are being adopted in management accounting. He finds take-up well below anticipated levels and provides a range of explanations as to why this has been the case.

The Journal continues to invite a wide range of contributions, and we are always interested in new proposals on theme issues.

\section{Forthcoming papers include}

Learning from abandoned IS development projects. Kweku Ewusi-Mensah and Zbigniew Przasnyski.

Coping with information technology? How British executives perceive the key IS management issues in the mid-1990s. Bob Galliers, Yasmin Merali and Laura Spearing.

Information technology enabled change: the risks and rewards of business process redesign and automation. Kirk Fiedler, Varun Grover and James Teng.

Introducing EIS into organizations: separating fact from fallacy. Brian Fitzgerald and Ciaran Murphy.

Matching an IT project with an appropriate method of evaluation: a research note. Barbara Farbey, Frank Land and David Targett.

Implementing multiple tutoring strategies in an intelligent tutoring system for music learning. Marios Angelides and Amelia Tong.

A methodology for the evaluation of information technology for strategic implementation. Steve Elliot. 